Eur Neurol 2002;47:58-61

\title{
Massive Four-Teritories Stroke: Bilateral Middle and Anterior Cerebral Artery Infarctions
}

Gerhard F. Hamann a, Ilonka Eisensehr ${ }^{\mathrm{a}}$, Thomas Mayer ${ }^{\mathrm{b}}$ Martin Liebetrau ${ }^{\mathrm{a}}$

Departments of a Neurology and ${ }^{\mathrm{b}}$ Neuroradiology, Ludwig Maximilians University, Klinikum Grosshadern, Munich,

Germany

\section{Introduction}

Multiple brain infarction is seen in a limited number of acute strokes. For example, in the Lausanne stroke register, only 40 of 751 patients with acute first-time stroke of the anterior circulation demonstrated multiple infarctions [1]. Only 10 of the 40 patients with multiple brain infarction showed bilateral infarctions, related either to cardioembolism or to large artery disease [1]. Using diffusionweighted sequences (DWI), magnetic resonance imaging (MRI) can evaluate the age of each lesion, determining whether these multiple brain infarcts occurred simultaneously or singly [2]. Using this sensitive technique, it is evident that the rate of multiple infarctions could be higher than previously suspected. In another study, among 142 patients with acute infarcts, 43 had more than one lesion on diffusion-weighted MRI [3]. From these 43 patients, 13 had acute multiple infarctions and 6 had multiple new and old strokes. Thus, about $15 \%$ of all patients experienced acute multiple brain infarctions in this patient group [3]. This rate is substantially higher than previously reported 5\% [1]. Here, we report a most striking case of multiple brain infarctions involving four cerebral artery territories.

\section{Case Report}

A woman aged 85 years was admitted to our acute stroke unit with motor problems and sensory deficit in the left arm. The neurological examination revealed a left-sided paresis of the arm and facial paresis. A history of atrial fibrillation and coronary heart disease was noted. The initial MRI examination detected a small lesion on DWI in the right internal capsule. Less than $1.5 \mathrm{~cm}$ in diameter, and located in the lenticulostriatal territory, this lesion was judged to be a lacunar infarction (fig. 1b). Multiple older white matter lesions seemingly confirmed this pathophysiological concept. Ultrasonographic investigations depicted a stenosis of the right internal carotid artery (ICA) in the carotid siphon area. Magnetic resonance angiography (MRA) confirmed this discovery, showing a signal reduction in the $\mathrm{C} 1$ and $\mathrm{C} 2$ segment of the right ICA. The MRA of the circulus of Willis revealed the lack of posterior communicating arteries on both 

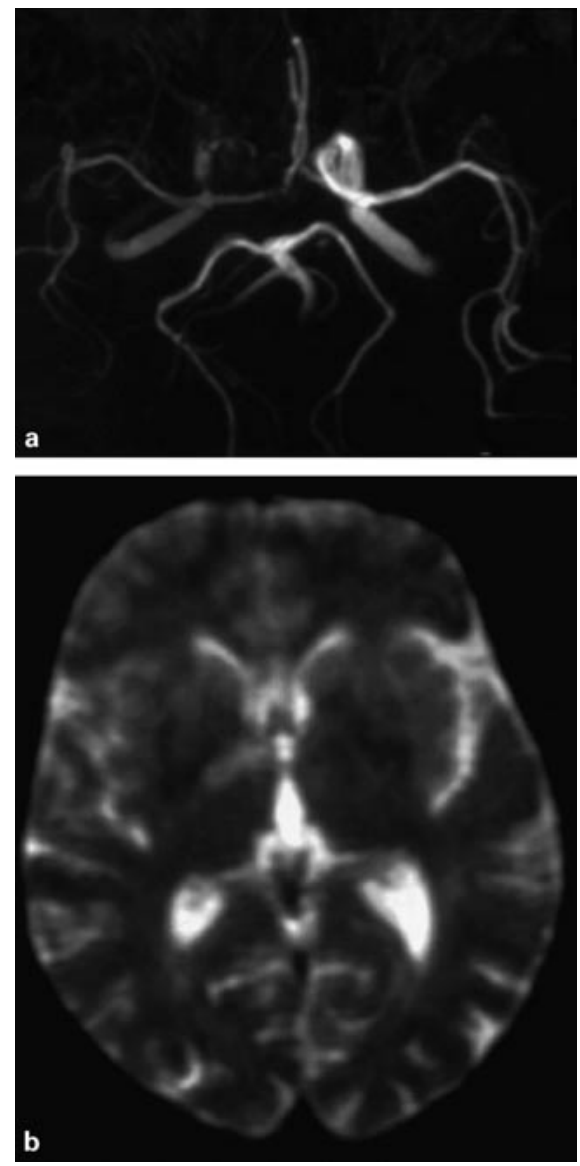

Fig. 1. MRI and MRA from the initial investigation (day 1) of our patient following her admission with slight hemiparesis of the left arm. a MRA shows the signal loss in the right ICA in the $\mathrm{C} 1$ and $\mathrm{C} 2$ segment. It is also obvious that both posterior communicating arteries are absent. b MRI shows a small signal hyperdensity in the right internal capsule at the border zone to the thalamus.

sides (fig. 1a). On day 2 after admission, the neurological deficit had improved and the patient was apparently symptom free. The neurological examination at this time showed only a slight paresis on the left side without other symptoms. On day 3 , the patient suddenly lost consciousness. She developed a deep coma without reactivity to painful stimuli and a progressive loss of brain stem reactivity.

An MRI on the third day detected bilateral signal intensities on DWI in the territories of the middle cerebral artery (MCA) and anterior cerebral artery (ACA) (fig. 2a). On the MRA, both ICAs were occluded (fig. 3). The posterior part of the MCA was still partly perfused, which was proven by MR perfusion images. On the fourth day, the T2 images also showed extensive infarcts in both ACA and MCA territories, sparing the posterior part of both MCA territories (fig. $2 b)$. The patient never regained consciousness and died on the fourth day with signs of transtentorial herniation.

The pathological examination showed a small thrombus in the right ICA. The left ICA was recanalized, and no thrombus in the heart or aorta could be found.

\section{Discussion}

The patient developed a small infarct in the right internal capsule due to either a lacunar syndrome, large artery disease of the carotid artery on the right side or atrial fibrillation. The initial MRA showed a stenosis of the right ICA in the $\mathrm{C} 1$ and $\mathrm{C} 2$ segment, which ultrasound techniques also proved. Both posterior communicating arteries were aplastic, and most of the carotid artery territories in both hemispheres were supplied by the left ICA. The initial MRA and ultrasound investigations demonstrated a normal left ICA without stenosis. The sudden deterioration of the patient, 3 days after the initial symptoms, suggested a basilar artery occlusion. The MRI proved that the basilar artery was open and occlusion of the left ICA had produced a severe forebrain ischemia in both hemispheres. The transient occlusion of the left ICA was probably caused by an acute embolic mechanism on the basis of the underlying atrial fibrillation. Both the negative MRA and ultrasound investigations before the left ICA occlusion and the open vessel in the pathological examination support this hypothesis. After this, the patient experienced a complete forebrain ischemia, which is produced experimentally by ligation of both ICAs in rats and gerbils, causing an ideal situation to study the effects of global ischemia and the amelioration of that devastating state [4]. Taking into account the diverse anatomical structures and physiologies of the animals studied, pathological examination does suggest striking similarities [5]. The anomalousness of the circulus of Willis with aplasia of both communicating posterior arteries was the anatomic substrate which allowed the development of these extensive bilateral lesions in our patient. The highgrade stenosis of the right ICA was the second prerequisite which allowed the embolic occlusion of the left ICA to produce a nearly complete carotid artery territory stroke in both hemispheres. The salvage of the posterior MCA territories on both sides could be explained by leptomeningeal anastomosis from the posterior cerebral arteries. Previous reports on bilateral ICA disease refer mainly to chronic arterial occlusive disease with rather few neurological changes [6]. In a French group of 19 patients with bilateral ICA occlusion, only minor neurological symptoms were noted. In young persons, even acute bilateral ICA occlusion may result in minor neurological symptoms. In one report, a young patient was reported to have suffered from bilateral ICA occlusion by a blunt trauma with only a narrow visual field deficit [7]. Just one study from Japan reports a 68 -year-old woman who experienced sudden onset of coma with bilateral internal carotid occlusion by cardioembolism. This patient apparently suffered from a similar infarction type to that reported here, even though the exact lesion location and size was not detailed [8].

In the opinion of most stroke neurologists, multiple brain infarction is likely caused by cardioembolism; however, recent Korean evidence suggests that mainly large vessel disease causes multiple brain infarctions in the anterior circulation [9]. In the instances of Yamaguchi et al. [8], our current case and other severely affected patients, a cardioembolic source plus large vessel disease might contribute to the pathophysiological background. Various other mechanisms may also be involved in multiple brain infarctions, such as paraneoplastic coagulation abnormalities, infections and various diseases [1, 10].

We conclude that total forebrain ischemia in humans is a rare but devastating condition. Prerequisites can be assumed, such as severe carotid stenosis, absence of a functional collateral system via the circulus of Willis and the combination of large artery disease and a cardioembolic source. The clinical presentation may mimic the onset of acute basilar occlusion. 
Fig. 2. a Diffusion-weighted MRI on the third day reveals the diffusion deficit in both ACA and most of both MCA territories. b The T2 images from the MRI on the fourth day show a similar distribution to that previously noted on DWI.

Fig. 3. MRA after the onset of coma on day 3 shows a loss of signal in both ICA territories. Note the absence of any carotidal vessels in the intracranial portion.
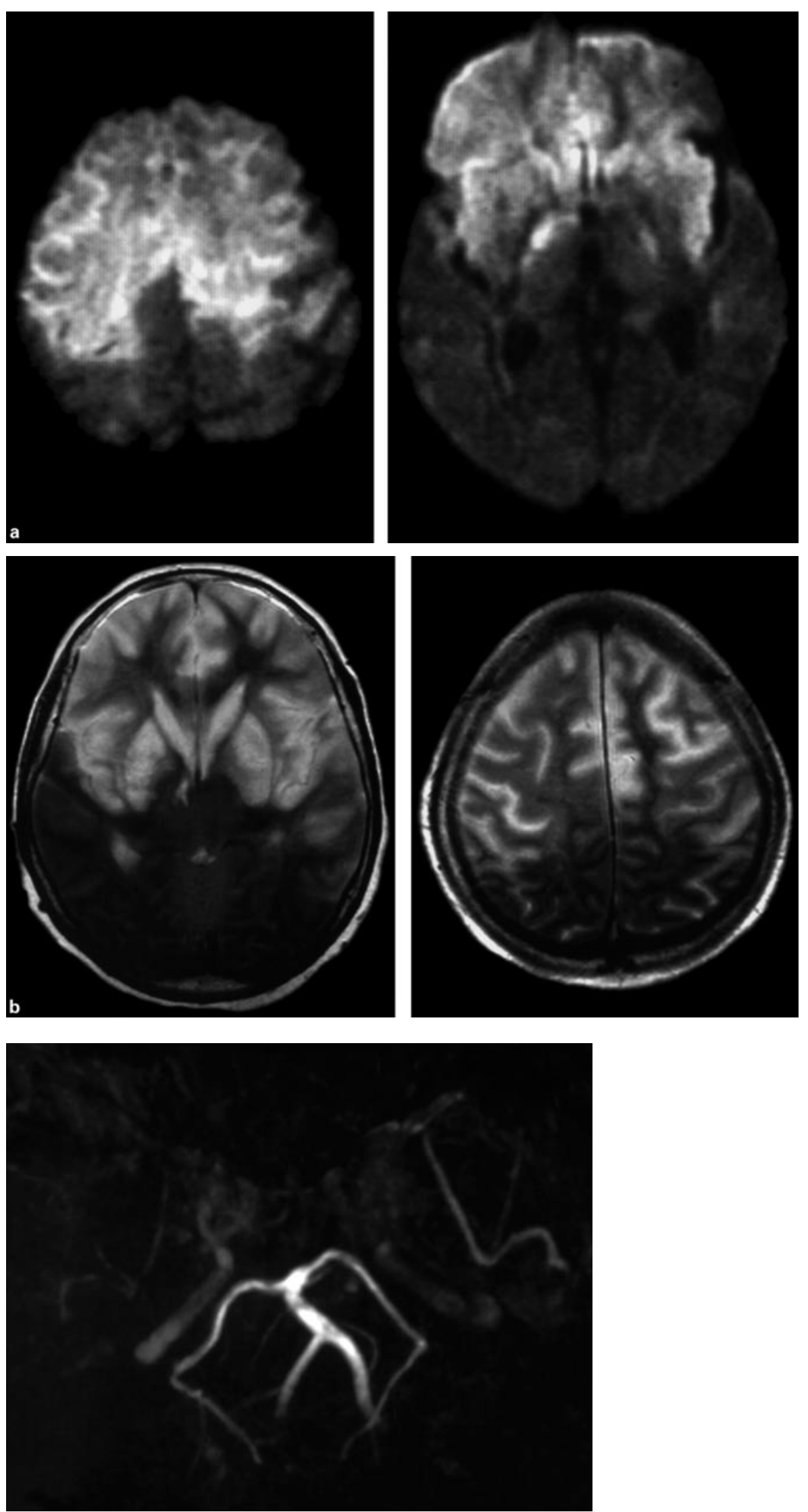


\section{References}

1 Bogousslavsky J, Bernasconi A, Kumral E: Acute multiple infarction involving the anterior circulation. Arch Neurol 1996;53:50-57.

2 Fitzek C, Tintera J, Muller-Forell W, Urban P, Thomke F, Fitzek S, Hopf HC, Stoeter P: Differentiation of recent and old cerebral infarcts by diffusion-weighted MRI. Neuroradiology 1998;40:778-782.

3 Altieri M, Metz RJ, Muller C, Maeder P, Meuli R, Bogousslavsky J: Multiple brain infarcts: Clinical and neuroimaging patterns using diffusionweighted magnetic resonance. Eur Neurol 1999;42:76-82.

4 Dirnagl U, Thoren P, Villringer A, Sixt G, Them A, Einhaupl KM: Global forebrain ischaemia in the rat: Controlled reduction of cerebral blood flow by hypobaric hypotension and two-vessel occlusion. Neurol Res 1993;15: 128-130.

5 Ogata J, Fujishima M, Morotomi Y, Omae T: Cerebral infarction following bilateral carotid artery ligation in normotensive and spontaneously hypertensive rats: A pathological study. Stroke 1976;7:54-60.

6 Catala M, Rancurel G, Raynaud C, Leder S, Kieffer E, Koskas F: Bilateral occlusion of the internal carotid arteries. Analysis of a series of 19 patients (in French). Rev Neurol (Paris) 1995;151:648-656.

7 Sadun AA, Sebag J, Bienfang DC: Complete bilateral internal carotid artery occlusion in a young man. J Clin Neuroophthalmol 1983;3:63-66.

8 Yamaguchi S, Oki S, Ogasawara H, Sato H, Ito Y, Hibino S: A case of simultaneous bilateral carotid occlusion (in Japanese). No Shinkei Geka 1997;25:247-251.

9 Roh JK, Kang DW, Lee SH, Yoon BW, Chang KH: Significance of acute multiple brain infarction on diffusion-weighted imaging. Stroke 2000;31: 688-694.

10 Vassallo R, Remstein ED, Parisi JE, Huston J, Brown RD: Multple cerebral infarctions from nonbacterial thrombotic endocarditis mimicking cerebral vasculitis. Mayo Clin Proc 1999;74:798-802.

Prof. Dr. Gerhard Hamann, Department of Neurology Ludwig Maximilians University, Klinikum Grosshadern Marchioninistrasse 15, D-81377 Munich (Germany)

Tel. +49 897095 3670, Fax +49 8970953677

E-Mailhamann@brain.nefo.med.uni-muenchen.de 